\title{
Synthesis of copper nanoparticles in polycarbonate by ion implantation
}

\author{
ANNU SHARMA*, SUMAN BAHNIWAL, SANJEEV AGGARWAL, S CHOPRA ${ }^{\dagger}$ and \\ D KANJILAL ${ }^{\dagger}$ \\ Department of Physics, Kurukshetra University, Kurukshetra 136 119, India \\ ${ }^{\dagger}$ Inter University Accelerator Centre, Aruna Asaf Ali Marg, New Delhi 110 067, India
}

MS received 16 July 2009; revised 12 October 2009

\begin{abstract}
Copper nanoparticles have been synthesized in polycarbonate by $75 \mathrm{KeV} \mathrm{Cu}^{-}$ion implantation with various doses ranging from $6.4 \times 10^{15}$ to $1.6 \times 10^{17}$ ions $/ \mathrm{cm}^{2}$ with a beam current density of $800 \mathrm{nA} / \mathrm{cm}^{2}$. The composites formed were structurally characterized using Ultraviolet-Visible (UV-Visible) absorption spectroscopy. The appearance of particle plasmon resonance peak, characteristic of copper nanoparticles at $603 \mathrm{~nm}$ in absorption spectra of polycarbonate implanted to a dose of $1.6 \times 10^{17} \mathrm{ions} / \mathrm{cm}^{2}$, indicates towards the formation of copper nanoparticles in polycarbonate. Transmission electron microscopy further confirms the formation of copper nanoparticles having size $\sim 3 \cdot 15 \mathrm{~nm}$. The formation of copper nanoparticles in the layers carbonized by $\mathrm{Cu}^{-}$implantation has been discussed. The synthesized copper-polycarbonate nanocomposite has been found to be more conducting than polycarbonate as ascertained using current-voltage characteristics.
\end{abstract}

Keywords. Polycarbonate; nanoparticles; ion implantation; UV-Visible spectroscopy.

\section{Introduction}

Nanocomposite materials containing nanometer-size metallic particles embedded in polymers have recently generated increasing interest as advanced technological materials because they show attractive optical properties at the surface plasmon resonance (SPR) wavelength due to dielectric and quantum confinement effects. When these nanocomposite materials with certain metallic nanoparticles like copper, gold and silver are exposed to light they exhibit a strong absorption maximum, at a particular wavelength called the SPR wavelength, due to photon-induced oscillation of the electron charge density. Such pronounced resonance lines shown by noble-metal nanoparticles caused by collective excitations of conduction electrons are also known as particle plasmon resonance (PPR) or Mie plasmons (Kreibig and Vollmer 1995). In general, the band width, intensity and position of the PPR wavelength of these metallic particles vary with particle size and shape and with their surrounding matrix (Kreibig and Vollmer 1995; Beecroft and Ober 1997; Lance et al 2003). The sensitivity of the PPR frequency to small variations of these parameters can be used in various applications such as colour filters (Quinten 2000; Biswas et al 2004) sensors (Haes and Van

*Author for correspondence (talk2annu@gmail.com)
Duyne 2002; Haes et al 2004) and all optical switching devices (Stegeman and Wright 1990).

Probing the particle-matrix interface physics is not an experimentally easy task and little knowledge exists on the subject. On the other hand, the contribution of the particle-matrix interaction may be important for the tailoring of the material physical properties. As a consequence of small dimensions of nanoparticles, quantumsize effects arise which introduce changes in its optical properties compared to the bulk material. Because of the relatively large number of atoms that conforms a metallic nanoparticle and its shape, these quantum effects are difficult to calculate. Thereby, having experimental evidence of such effects is crucial in terms of volume concentration and their spatial distribution.

Several methods such as co-sputtering (Schurmann et al 2005), reduction of a metal salt (Troger et al 1997; Huang and Yang 1999) and ion implantation (Umeda et al 2003; Boldyryeeva et al 2005; Yuguang et al 2005; Teixeira et al 2009) are used to produce these composite systems. Among them, ion implantation stands out for its numerous advantages. Precise concentration control and in-depth dopant profiling is achieved by designing the implantation parameters, while surface localization is resolved using masks or focused ion beam techniques. These possibilities are particularly important for the application of the technique of ion beam synthesis to produce new compounds and different phases in the irra- 
diated polymer surfaces, yielding new interesting properties, by implanting ions of various chemical elements (Umeda et al 2003; Boldyryeeva et al 2004; Stepanov 2004; Takeda et al 2004; Boldyryeeva et al 2005; Yuguang et al 2005; Srivastava et al 2008; Teixeira et al 2009).

In the present work, copper nanoparticles have been synthesized inside the polycarbonate matrix by low energy ion implantation and their effects on optical as well as electrical properties have been discussed. Polycarbonate was chosen as the polymer matrix because it is highly transparent in the visible spectral range, which makes it an excellent substitute of glass substrate in a variety of optical and optoelectronics applications such as flat panel displays, antireflection coatings, solar cells and heat mirrors (DeRudder 2000). Polycarbonate is also highly sensitive to ion irradiation, and is often employed as a resist for electron beam and ion beam lithography and is thus well suited to serve as packaging material for medical disposables which are slated to be radiation sterilized.

\section{Experimental}

Polycarbonate samples of area $2 \times 2 \mathrm{~cm}^{2}$ were cut from its $250 \mu \mathrm{m}$ thick, commercially available and optically transparent sheet. Samples were implanted with $75 \mathrm{KeV}$ $\mathrm{Cu}^{-}$ions to various doses ranging from $6.4 \times 10^{15}$ to $1.6 \times 10^{17} \mathrm{ions} / \mathrm{cm}^{2}$ at a beam current density of $\sim 800 \mathrm{nA} / \mathrm{cm}^{2}$ under a vacuum of $\sim 10^{-7}$ Torr using low energy ion beam facility at Inter University Accelerator Centre (IUAC), New Delhi. The optical absorption and transmission measurements of all the specimens were performed using a Shimadzu double beam double monochromator spectrophotometer (UV-2550) equipped with an integrating sphere assembly ISR-240A in the wavelength range of 190-900 nm with a resolution of $0.5 \mathrm{~nm}$. All these absorption spectra were recorded keeping air as the reference.

The size of the copper nanoparticles embedded in polycarbonate was determined by transmission electron microscopy (TEM) using Technai G20-Stwin Trwas electron microscope operating at $200 \mathrm{kV}$ at Indian Institute of Technology, New Delhi. The current-voltage (IV) characteristics of the formed nanocomposite were measured using Keithley (6517A) Electrometer at room temperature. The samples were laid between the two electrodes and a testing voltage of $10 \mathrm{~V}$ was applied.

\section{Results and discussions}

The optical absorption spectra of the pristine and $75 \mathrm{KeV}$ $\mathrm{Cu}^{-}$ion-implanted polycarbonate samples with various doses are shown in the figure 1. Absorption peaks in UV region are due to electronic transitions between occupied and unoccupied molecular orbitals and the spectrum in this region is particularly sensitive to conjugated bonds. The strongest peaks in the UV are generally due to $\pi-\pi^{*}$ transitions which are likely to be associated with the phenyl groups in polycarbonate (Pavia et al 1994). The $\pi-\pi^{*}$ peak is known to shift to higher wavelengths as conjugation is increased. The absorption spectra of the virgin polycarbonate (curve a, figure 1) show minimum absorption. As a result of copper ion implantation to a dose of $6.4 \times 10^{15}$ ions $/ \mathrm{cm}^{2}$ (curve b, figure 1) the absorption increases. With a further increase in dose to $1.2 \times 10^{16}$ ions $/ \mathrm{cm}^{2}$ the absorption continues to increase (curve c, figure 1). At a dose of $1.6 \times 10^{17} \mathrm{ions} / \mathrm{cm}^{2}$ (curve d, figure 1) along with the increase in absorption, PPR peak, characteristic of copper nanoparticles, centred at $603 \mathrm{~nm}$ is also observed (Umeda et al 2003; Yuguang et al 2005). The presence of the PPR peak is a good indicator of the formation of copper nanoparticles inside the polycarbonate matrix.

The origin of the resonance is attributed to the coupling between the oscillation of the conduction band electrons cloud on the surface of the copper nanoparticles and the incident electromagnetic wave in the quasistatic regime (Kreibig and Vollmer 1995). For particles of diameter less than $\lambda / 20$, the optical absorption coefficient $\alpha$ of the composite is given by (Kreibig and Vollmer 1995):

$$
\alpha\left(\mathrm{cm}^{-1}\right)=\left(18 \pi V n^{3} \varepsilon_{2}\right) /\left(\lambda\left(\varepsilon_{1}+2 n^{2}\right)^{2}+\varepsilon_{2}^{2}\right),
$$

where $\alpha$ is the absorption coefficient of a material of refractive index $n$ embedded with metal spheres of volume fraction $V$, with radii small compared to the wavelength $\lambda$ of the incident light. $\varepsilon_{1}$ and $\varepsilon_{2}$ are the frequency-dependent real and imaginary components of the metal particle, calculated from the optical constants of their bulk metal

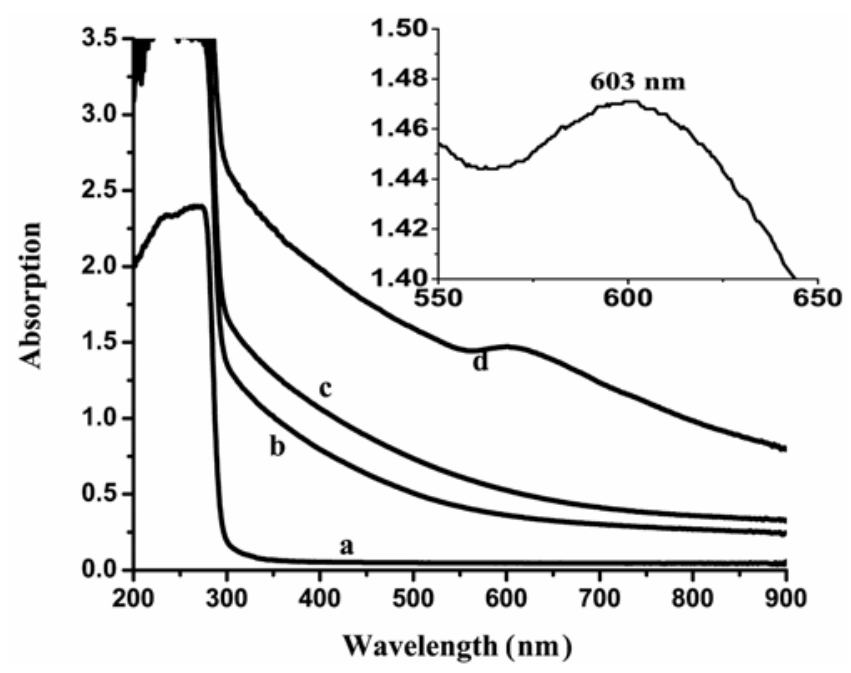

Figure 1. UV-Visible absorption spectra of polycarbonate (a) unimplanted and implanted with $75 \mathrm{KeV}$ to a dose of (b) $6.4 \times$ $10^{15} \mathrm{Cu}^{-} / \mathrm{cm}^{2}$, (c) $1.2 \times 10^{16} \mathrm{Cu}^{-} / \mathrm{cm}^{2}$ and (d) $1.6 \times 10^{17} \mathrm{Cu}^{-} /$ $\mathrm{cm}^{2}$. (Inset: absorption spectra from 550 to $650 \mathrm{~nm}$ of polycarbonate implanted to a dose of $1.6 \times 10^{17} \mathrm{Cu}^{-} / \mathrm{cm}^{2}$.) 
(Palik 1985). The absorption coefficient has its maximum value when $\left(\varepsilon_{1}+2 n^{2}=0\right)$, which is the condition for the PPR. Thus PPR frequency depends on the dielectric function as well as the metal particle size and composition.

The sizes of copper nanoparticles are calculated according to the following formula, assuming the free particle behaviour of the conduction electrons (Arnold 1975):

$$
d=2 h v_{\mathrm{f}} / \Delta E_{1 / 2}
$$

where $d$ is the diameter of the copper nanoparticles, $v_{\mathrm{f}}$ $\left(1.57 \times 10^{6} \mathrm{~m} / \mathrm{s}\right)$ is the Fermi velocity of electrons in bulk copper, $h$ is Planck's constant, and $\Delta E_{1 / 2}$ is the FWHM of the absorption band. The above equation is valid as long as the copper nanoparticles dimension is smaller than the mean free path of the electrons in the bulk metal. The mean free path of electrons is about $40 \mathrm{~nm}$ at room temperature for bulk copper. Using (2), size of copper nanoparticles comes out to be $\sim 3.06 \mathrm{~nm}$.

The growth of copper nanoparticles in the sample implanted with $1.6 \times 10^{17}$ ions $/ \mathrm{cm}^{2}$ was further investigated by TEM. Figure 2(a) shows the TEM micrograph of the
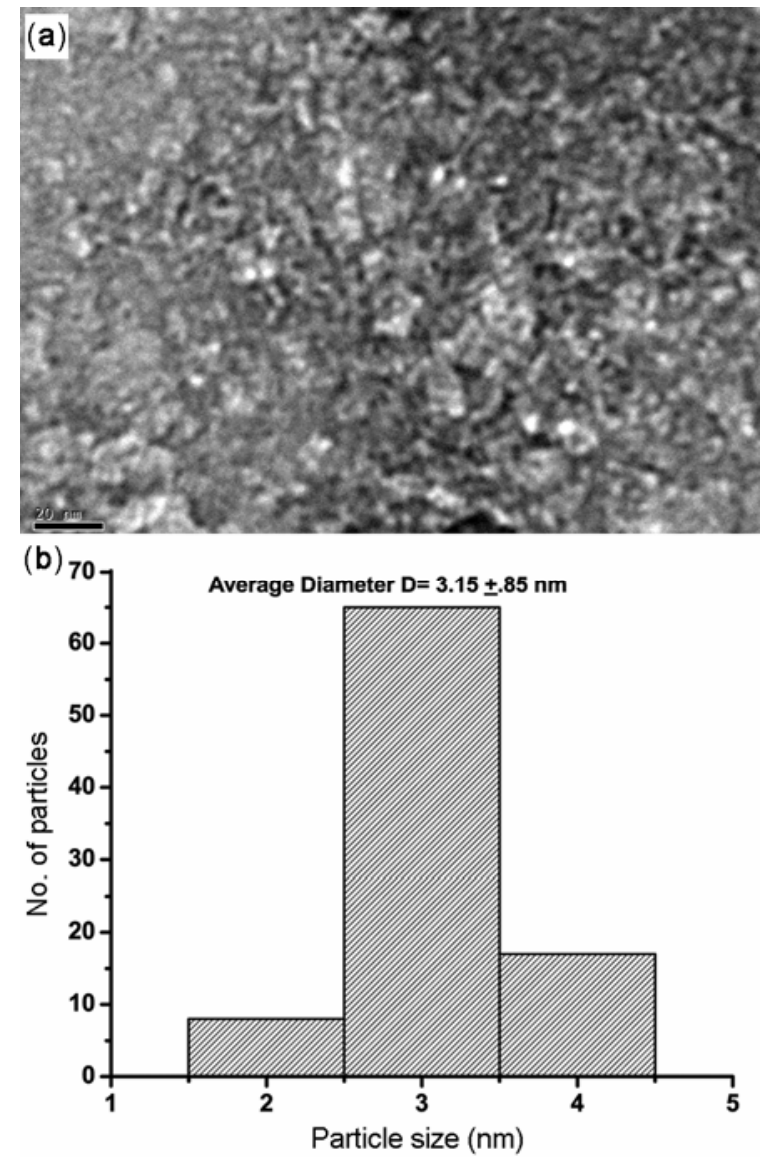

Figure 2. (a) TEM micrograph of the copper-polycarbonate nanocomposite synthesized at an implantation dose of $1.6 \times$ $10^{17} \mathrm{Cu}^{-} / \mathrm{cm}^{2}$. (b) Size distribution of copper nanoparticles obtained from figure 2(a). sample implanted with $1.6 \times 10^{17}$ ions $/ \mathrm{cm}^{2}$. The size distribution of these copper nanoparticles has been presented in figure 2(b). The average size of the nanoparticles comes out to be $3 \cdot 15 \pm 0 \cdot 85 \mathrm{~nm}$, which agrees well with the size calculated using UV-Visible spectroscopy.

The transmission spectra of the unimplanted and $\mathrm{Cu}^{-}$ implanted polycarbonate is shown in figure 3 . For virgin polycarbonate (curve a, figure 3 ) transmission is $~ 90 \%$ in the entire visible region and falls sharply thereafter becoming opaque in the $\mathrm{UV}$ region. After $\mathrm{Cu}^{-}$ion implantation to a dose of $6.4 \times 10^{15}$ ions $/ \mathrm{cm}^{2}$ (curve b, figure 3 ) it falls to $60 \%$ at $900 \mathrm{~nm}$ and then decreases continuously up to $4 \%$ at $300 \mathrm{~nm}$. With further increase in ion dose to $1.2 \times 10^{16}$ ions $/ \mathrm{cm}^{2}$ (curve c, figure 3 ) transmission falls to $45 \%$ at $900 \mathrm{~nm}$ and then decreases continuously up to $\sim 2 \%$ at $300 \mathrm{~nm}$. Transmission falls abruptly and reaches in the range of $0-4 \%$ in the visible region at a highest implantation dose of $1.6 \times 10^{17}$ ions $/ \mathrm{cm}^{2}$ (curve $\mathrm{d}$, figure 3 ).

The continuous decrease of transmission with increase in ion dose as discussed above can be correlated with the sharp change in colour of the implanted polycarbonate specimens from transparent (virgin) to brown at a dose of $6.4 \times 10^{15}$ ions $/ \mathrm{cm}^{2}$ and finally dark brown at a highest implantation dose of $1.2 \times 10^{17}$ ions $/ \mathrm{cm}^{2}$. The change in colour can be due to dehydrogenation or formation of colour-sensitive conjugated bonds with loosely bound $\pi$-electrons which can easily be excited by the visible photons leading to observed change in colour of the implanted surface (Pavia et al 1994).

In the present case of implantation by $\mathrm{Cu}^{-}$ions at relatively low energy $75 \mathrm{KeV}$, nuclear collisions prevail in ion-insulator interactions. They displace atoms in the polymer matrix and break some of chemical bonds in it.

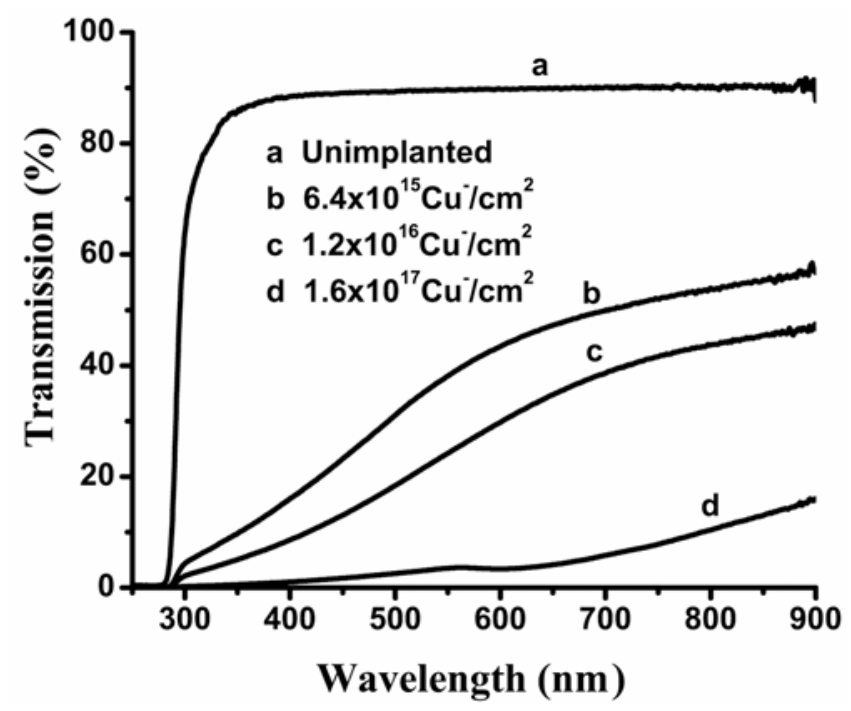

Figure 3. The UV-Visible transmission spectra of the polycarbonate (a) unimplanted and implanted with $75 \mathrm{KeV}$ to a dose of (b) $6.4 \times 10^{15} \mathrm{Cu}^{-} / \mathrm{cm}^{2}$, (c) $1.2 \times 10^{16} \mathrm{Cu}^{-} / \mathrm{cm}^{2}$ and (d) $1.6 \times 10^{17} \mathrm{Cu}^{-} / \mathrm{cm}^{2}$. 
In principle, copper atoms may combine with arising organic radical and polymer ions or take part in the oxidation reactions. However, because of a great difference in Gibbs free energy between copper atoms and atoms of polycarbonate elements, $\mathrm{Cu}-\mathrm{Cu}$ bonding is energetically favourable. The formation of metal nanoparticles proceeds in several stages: the accumulation and subsequent supersaturation of copper atoms in a local surface region of the polymer, the formation of nuclei consisting of several atoms and the growth of copper particles from such nuclei assuming that the nanoparticles nucleate and grow via the successive attachment of copper atoms. Thus it can be concluded that this process is simultaneously governed by the diffusion coefficient and local concentration of copper atoms (Umeda et al 2003; Yuguang et al 2005).

From figures 1 and 3 it is clear that as a result of $\mathrm{Cu}^{-}$ ion implantation the absorption increases and the absorption edge shifts to longer wavelengths; the shifting of the onset of absorption to longer wavelength has been interpreted as an indicator of the formation of carbonaceous clusters or networks of conjugated unsaturated bonds (Zhu et al 2002). During implantation, hydrogen, low molecular hydrocarbons, $\mathrm{CO}, \mathrm{CO}_{2}$ and other gases are released from polymeric materials, which cause the enrichment of carbon atoms leading to the formation of hydrogenated amorphous carbon (Zhu et al 2002; Stepanov 2004; Takeda et al 2004; Srivastava et al 2008).

Thus the implantation of copper not only generates radiation-induced defects but also causes the nucleation and growth of metal nanoparticles. As a result, along with absorption intensity variation, an absorption band associated with copper nanoparticles is also observed.

Figure 4 shows the current-voltage plots of synthesized copper-polycarbonate nanocomposite at the implantation dose of $1.6 \times 10^{17} \mathrm{Cu}^{-} / \mathrm{cm}^{2}$ along with unimplanted polycarbonate. It can be discerned from figure 4 that

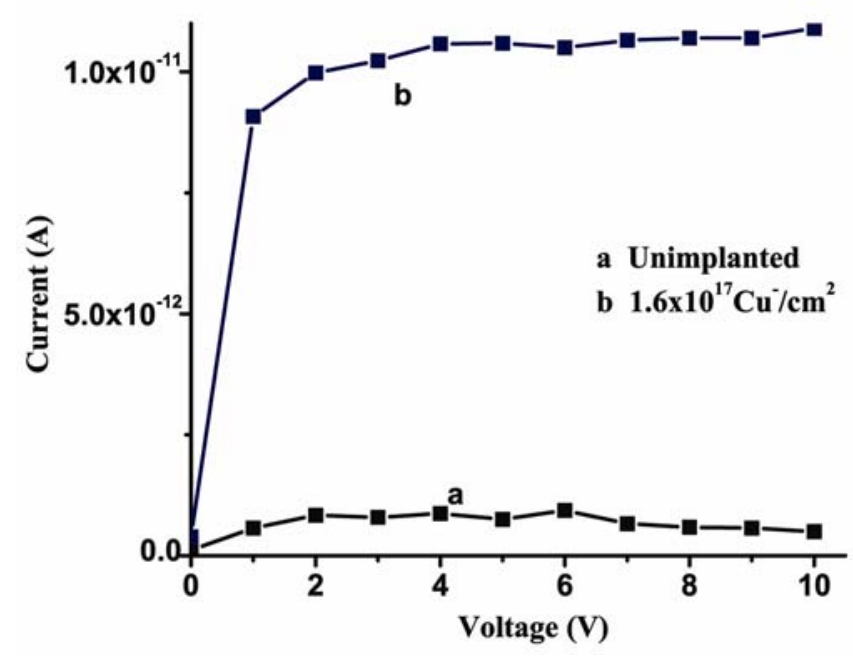

Figure 4. The $I-V$ characteristics of (a) unimplanted polycarbonate and (b) copper-polycarbonate nanocomposite synthesized at an implantation dose of $1.6 \times 10^{17} \mathrm{Cu}^{-} / \mathrm{cm}^{2}$. unimplanted polycarbonate behaves as a perfect insulator, with the embedding of copper nanoparticles of size $\sim 3 \mathrm{~nm}$ in polymer matrix the resistance of the resulting composite decreases. The electrical properties of nanocomposite films depend upon their microstructure and are strongly linked to the particles nanostructure, particularly to the distance between the particles (Bloor et al 2005). The resistance of copper-polycarbonate nanocomposite synthesized at the dose of $1.6 \times 10^{17} \mathrm{Cu}^{-} / \mathrm{cm}^{2}$ decreases, because at such a high dose the separation between the nanoparticles is reduced. With smaller distance between nanoparticles, the electrical field induced is large enough to cause the electrons to tunnel from one nanoparticle to a neighbouring one. Consequently conduction occurs via field-induced tunneling and results in the decrease in resistance. The understanding of the electrical behaviour of such nanocomposites can be used to prepare sensors or other devices with a well-defined conductivity.

\section{Conclusions}

Thus, negative ion implantation is a powerful method of fabrication of copper nanoparticles in polycarbonate matrix. When low energy $\mathrm{Cu}^{-}$ions are implanted into polycarbonate matrix, besides the increase in absorption intensity with increased dose, an absorption band, characteristic of copper nanoparticles is observed around $603 \mathrm{~nm}$ at the dose of $1.6 \times 10^{17}$ ions $/ \mathrm{cm}^{2}$. This indicates the formation of copper nanoparticles in polycarbonate matrix. TEM further confirms the formation of copper nanoparticles of size $3 \cdot 15 \mathrm{~nm}$. The I-V characteristics of copper-polycarbonate composites indicate that resistance decreases with increasing copper concentration.

\section{Acknowledgements}

The authors are thankful to Department of Science and Technology (DST), New Delhi, India for providing kind support. We are thankful to Ms Devyani, Inter University Accelerator Centre, Aruna Asaf Ali Marg, New Delhi, India for her kind help during implantation. Authors are also grateful to Dr Vidyanand, IIT, New Delhi for performing TEM.

\section{References}

Arnold G W 1975 J. Appl. Phys. 464466

Beecroft L L and Ober C K 1997 Chem. Mater. 91302

Biswas A, Aktas O C, Kanzow J, Saeed U, Strunkus T, Zaporjtchenko V and Faupel F 2004 Mater. Lett. 58 1530

Bloor D, Donnelly K, Hands P J, Laughlin P and Lussey D 2005 J. Phys. D. Appl. Phys. 382851

Boldyryeeva H, Umeda N, Plakin O A, Takeda Y and Kisimoto N 2005 Surf.\& Coat. Technol. 196373 
Boldyryeva H, Kishimoto N, Umeda N, Kono K, Plaksin O A and Takeda Y 2004 Nucl. Instrum. Meth. Phys. Res. B219220953

DeRudder J L 2000 In Handbook of polycarbonate science and technology (eds) D G LeGrand and J T Bendler (New York: Marcel Dekker, Inc.)

Haes A J and Van Duyne R P 2002 J. Am. Chem. Soc. 124 10596

Haes A J, Zou S, Schatz G C and Van Duyne R P 2004 J. Phys. Chem. B108 109

Huang C and Yang C Z 1999 Appl. Phys. Lett. 741692

Kreibig U and Vollmer M 1995 Optical properties of metal clusters (Berlin: Springer)

Lance K K, Coronado E, Zhao L L and Schatz G C $2003 \mathrm{~J}$. Phys. Chem. B107 68

Palik E D 1985 Handbook of optical constants of solids (Academic Press)

Pavia D L, Lampman G M and Kriz G S 1994 Introduction to spectroscopy (New York: Harcourt Brace College) 2nd edn

Quinten M 2000 Appl. Phys. B: Lasers Opt. 73317
Schurmann U, Hartun W A, Takele H, Zaporojtchenko V and Faupel F 2005 Nanotechnology 161078

Srivastava S, Haridas M and Basu J K 2008 Bull. Mater. Sci. 31 213

Stegeman G I and Wright E M 1990 Opt. Quantum Electron. 22 95

Stepanov A L 2004 in Metal-polymer nanocomposites (eds) L Nicolais and G Carotenuto (Chichester: Wiley) pp. 241263

Takeda Y, Lu J, Okubo N, Plaksin O A, Suga T and Kishimoto N 2004 Vacuum 74717

Teixeira F S, Salvadori M C, Cattani M and Brown I G $2009 \mathrm{~J}$. Appl. Phys. 105064313

Troger L, Hunnefel H, Nunes S, Oehring M and Fritsch D 1997 J. Phys. Chem. B101 1279

Umeda N, Bandourko V V, Vasilets V N and Kishimoto N 2003 Nucl. Instrum. Meth. Phys. Res. B206 657

Yuguang W, Tonghe Z, Yawen Z, Gu Z, Huixing Z and Xiaoji Z 2005 Surf. \& Coat. Technol. 148221

Zhu Z, Sun Y, Liu C, Liu J and Jin Y 2002 Nucl. Instrum. Meth. Phys. Res. B193 271 